\title{
Los indicadores del manejo del régimen terapéutico y su relación con la evolución de la información adquirida durante el entrenamiento en diálisis peritoneal
}

\author{
Antonia Concepción Gómez Castilla - Ma Ángeles Ojeda Guerrero - Elena Carballo Pérez - Miguel Ángel Ramírez López - \\ Jesús Cárcamo Baena - Dolores Fernández Gordillo
}

Enfermera/o del Servicio de Nefrología

Hospital Virgen Macarena. Sevilla

\section{Resumen}

Los pacientes de diálisis peritoneal son sometidos a la intervención enfermera enseñanza individual para la obtención de conocimientos básicos, con el objetivo de efectuar el tratamiento en domicilio en condiciones de seguridad, que minimicen los riesgos que conlleva el proceso de diálisis peritoneal.

La adecuación de los objetivos esperados a las características y/o capacidad del paciente/familia, consigue individualizar las actividades propias de la intervención enseñanza, así como el nivel de conocimientos adquiridos.

Los indicadores relacionados con el manejo del proceso, la infección, la hidratación y la dieta, sirven para conocer el nivel de conocimientos del paciente/ familia, y así poder dar desde la unidad de diálisis peritoneal, apoyo y o seguimiento individualizado en el domicilio, según las necesidades del paciente.

La evolución del manejo del régimen terapéutico, es un hecho que siempre preocupa a la enfermería de diálisis peritoneal; el cumplimiento tanto de las normas aprendidas durante el entrenamiento como

Correspondencia:
Concepción Gómez Castilla
C/ Cañada de la viña nº 6
41927 Mairena del Aljarafe. Sevilla
E-mail: antoniac.gomez.sspa@juntadeandalucia.es

de los procedimientos descritos, son de suma importancia para minimizar las complicaciones intrínsecas al proceso terapéutico que nos ocupa.

La necesidad de establecer pautas para un reentrenamiento estructurado, hemos pretendido conocerla a través de los indicadores relacionados con los criterios de resultados que describimos a continuación:

- Conocimiento del manejo del régimen terapéutico (1813).

- Conducta de cumplimiento (1601).

- Control del riesgo (1902).

- Control de síntomas (1608).

Los criterios de resultados esperados corresponden al diagnóstico estandarizado en nuestro medio de 0082 Manejo efectivo del régimen terapéutico y 00162 Disposición para el manejo efectivo del régimen terapéutico, que son las etiquetas diagnósticas por excelencia al alta tras el entrenamiento para efectuar la diálisis peritoneal en el domicilio.

\section{PALABRAS CLAVE:}

- ENTRENAMIENTO DIÁLISIS PERITONEAL - DIAGNÓSTICOS ENFERMEROS

- INTERVENCIONES ENFERMERAS

- CRITERIOS DE RESULTADOS

- INDICADORES 
Therapeutic regimen management indicators and their relationship to the evolution of the information acquired during peritoneal dialysis training.

\section{Abstract}

Patients undergoing peritoneal dialysis receive individual training provided by nursing staff in order to obtain basic knowledge so that they can carry out the treatment at home in conditions of safety that minimize the risks involved in the peritoneal dialysis process.

Matching the desired goals to the characteristics and/or capacity of the patient/family individualizes the teaching activities and the level of knowledge acquired.

Indicators related to the management of the process, infection, hydration and diet serve to determine the level of knowledge of the patient/family, and thus provide support from the peritoneal dialysis unit and/ or individual monitoring at home, according to the patient's needs.

The evolution of therapeutic regimen management is always a matter that concerns peritoneal dialysis nursing staff; compliance both with the rules learned during training and with the procedures described is extremely important to minimize the intrinsic complications of the therapeutic process in question.

We have sought to determine the need to establish guidelines for structured retraining through the indicators related to the outcome criteria described below:

- Knowledge of therapeutic regimen management (1813).

- Compliance behaviour (1601).

- Risk control (1902).

- Symptom control (1608).

The expected outcome criteria correspond to the standardized diagnostic in our environment of 0082 Effective management of the therapeutic regimen and 00162 Readiness for effective management of the therapeutic regimen, which are the ideal diagnostic labels upon discharge from hospital after training to carry out peritoneal dialysis at home.

\section{KEY WORDS:}

- PERITONEAL DIALYSIS TRAINING

- NURSING DIAGNOSTICS

- NURSING INTERVENTIONS

- OUTCOME CRITERIA

- INDICATORS

\section{Introducción}

Los pacientes de diálisis peritoneal son sometidos a la intervención enfermera enseñanza individual para la obtención de conocimientos básicos, con el objetivo de efectuar el tratamiento en domicilio en condiciones de seguridad, que minimicen los riesgos que conlleva el proceso de diálisis peritoneal.

La adecuación de los objetivos esperados a las características y/o capacidad del paciente/familia, consigue individualizar las actividades propias de la intervención enseñanza, así como el nivel de conocimientos adquiridos $^{1,2,3,4,5,6,7}$.

Los indicadores relacionados con el manejo del proceso, la infección, la hidratación y la dieta, sirven para conocer el nivel de conocimientos del paciente/familia, y así poder dar desde la unidad de diálisis peritoneal, apoyo y o seguimiento individualizado en el domicilio, según las necesidades del paciente ${ }^{8}$.

La evolución del manejo del régimen terapéutico, es un hecho que siempre preocupa a la enfermería de diálisis peritoneal; el cumplimiento tanto de las normas aprendidas durante el entrenamiento como de los procedimientos descritos, son de suma importancia para minimizar las complicaciones intrínsecas al proceso terapéutico que nos ocupa.

Definiendo cumplimiento como "la medida en que el paciente asume las normas o consejos dados por el médico o personal sanitario, tanto desde el punto de vista de hábitos o estilo de vida recomendados, como del propio tratamiento farmacológico prescrito, expresándose con él, el grado de coincidencia entre las orientaciones dadas por el profesional y la realización por parte del paciente, realizadas estas tras una decisión completamente razonada, por este, abandonándose las connotaciones de sumisión que este término implicaba para el paciente" 
La necesidad de establecer pautas para un reentrenamiento estructurado, hemos pretendido conocerla a través de los indicadores relacionados con los criterios de resultados que describimos a continuación ${ }^{10}$.

\section{- Conocimiento del manejo del régimen terapéutico} (1813) definido como grado de comprensión sobre el régimen terapéutico especifico y los procedimientos, la dieta y prevención/control de la infección. Valorado mediante los siguientes indicadores: describe la dieta, describe la medicación y su uso, describe los procedimientos terapéuticos, describe las complicaciones potenciales, describe signos y síntomas de las complicaciones (medidos mediante los parámetros de medida de ninguno, escaso, moderado, sustancial y extenso).

- Conducta de cumplimiento (1601) definida como acciones basadas en el asesoramiento profesional para promocionar el bienestar, la recuperación y la rehabilitación. Valorado mediante los indicadores: comunica seguir pautas descritas, confianza en el profesional sanitario sobre la información recibida, conserva la cita, modifica la pauta orientada por el profesional sanitario, realiza las actividades de la vida diaria según prescripción.

- Control del riesgo (1902) definido como acciones para eliminar o reducir las amenazas para la salud; reales, personales y modificables. Valorado mediante los indicadores: modifica estilo de vida, reconoce factores de riesgo, reconoce cambios en el estado de salud, participa en la identificación sistemática de los riesgos identificados, desarrolla estrategias efectivas de control de riesgos.

- Control de síntomas (1608) definido como las acciones personales para minimizar los cambios adversos percibidos en el funcionamiento físico y emocional. Valorado mediante los indicadores: reconoce síntomas de sobrehidratación, reconoce síntomas de infección, utiliza signos de alarmas para buscar atención sanitaria, utiliza los recursos disponibles, utiliza medidas preventivas.

Los indicadores de cumplimiento, control del riesgo y control de síntomas han sido medidos mediante escala de Licker (valoraciones entre 1 y 5): nunca demostrado, raramente demostrado, a veces demostrado, frecuentemente demostrado y siempre demostrado ${ }^{11,12}$.
Los criterios de resultados esperados corresponden al diagnostico estandarizado en nuestro medio de 0082 Manejo efectivo del régimen terapéutico y 00162 Disposición para el manejo efectivo del régimen terapéutico, que son las etiquetas diagnosticas por excelencia al alta tras el entrenamiento para efectuar la diálisis peritoneal en el domicilio ${ }^{11,12}$.

\section{Objetivos}

Conocer la evolución en el tiempo de la información procesada durante el entrenamiento, manifestada por nivel de conocimientos, conducta de cumplimiento, control del riesgo y control de síntomas.

\section{Pacientes y método}

Realizamos estudio transversal descriptivo a 78 pacientes que habían estado en programa de diálisis peritoneal entre enero de 2004 y diciembre de 2009, con una edad media de 57,58 $\pm 15,97$ años (rango entre 26 y 84 ) y un tiempo en diálisis de $29,42 \pm 22,25$ meses (rango entre 3 y 92), de ellos 33 (42,3\%) eran hombres y $45(57,7 \%)$ eran mujeres. 53 pacientes $(67,9 \%)$ en DPA y $25(33,1 \%)$ en DPAC, a los que se había sometido a la intervención de enseñanza individual y guardaban en su historia datos relativos a la valoración de conocimientos adquiridos durante el entrenamiento.

Al termino del entrenamiento la etiqueta diagnostica habitual es manejo efectivo del régimen terapéutico y/o disposición para el manejo efectivo del régimen terapéutico. Nosotros medimos los indicadores correspondientes a los criterios de resultados esperados a partir de los 3 meses y posteriormente 1 ó 2 veces al año, habiendo utilizado para este estudio la ultima medición de cada paciente.

Los indicadores han sido agrupados correspondiendo el valor final al porcentaje de la evaluación de todos los indicadores valorados, de manera que el valor máximo posible que es el nivel de la escala 5 y se corresponde con el $100 \%$.

Hemos estudiado las siguientes variables: Edad, Sexo, tipo de diálisis, Tiempo en diálisis, Nivel de conocimientos adquiridos por el receptor del entrenamiento y los 
indicadores relacionados con los criterios de resultados: conocimientos sobre el manejo del régimen terapéutico, conducta de cumplimiento, control del riesgo y control de síntomas.

La fuente de datos ha sido la historia enfermera informatizada.

Análisis de datos con programa estadístico ssps 17.0 mediante frecuencias para variables cualitativas y medias y desviaciones para variables cuantitativas, analizando asociaciones entre variables mediante rho de spearman, test chi cuadrado

\section{Resultados}

El nivel de conocimientos adquiridos durante el entrenamiento se distribuye de la siguiente manera: Conocimientos extensos, lo que supone un nivel de conocimientos superior al $90 \%$ de los conocimientos evaluados lo adquirieron el $4 \%$ de los pacientes. Conocimientos sustanciales, definido por un nivel de conocimientos entre 71 y $90 \%$ de los conocimientos evaluados lo obtuvieron el $41 \%$ de los pacientes. En un nivel moderado (entre 51 y $70 \%$ ), lo obtuvieron otro $41 \%$ de los pacientes y con conocimientos escasos fueron valorados el $14 \%$ de los pacientes, lo que suponía haber obtenido una valoración entre el 31 y 50\% de los conocimientos evaluados, mientras no hubo ningún paciente que fuera valorado con conocimientos por debajo del $31 \%$.

El gráfico 1 representa el porcentaje de pacientes en cada nivel de conocimientos entre los indicadores obtenidos al alta de entrenamiento y los indicadores relacionados con el conocimiento del régimen terapéutico en el tiempo.

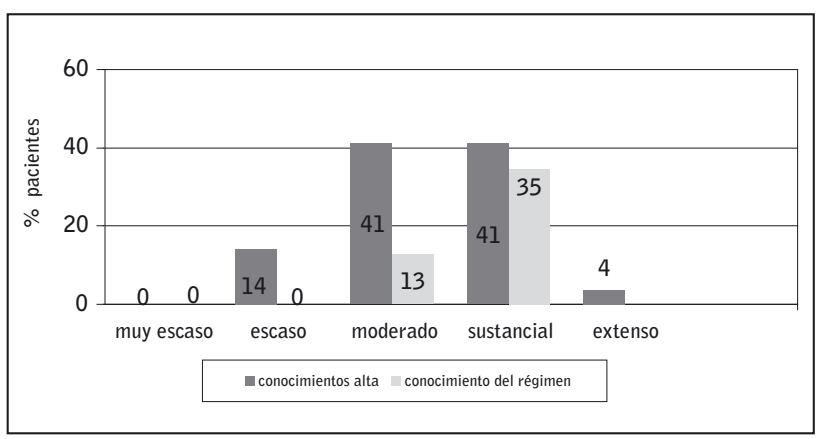

Gráfico 1: Evolución de los indicadores relacionados con el conocimiento terapéutico
Observamos que todos los pacientes han mejorado el nivel de conocimientos desde que fueron sometidos a la enseñanza individual para el manejo del régimen terapéutico, a la última medición de los indicadores implicados.

Al aplicar test de chi cuadrado se observan diferencias estadísticamente significativas en la evolución de estos indicadores ( $p$,000).

El gráfico 2 presenta la frecuencia relativa de los indicadores relacionados con el criterio de resultado conducta de cumplimiento, manteniendo la siguiente distribución: el 2,6\% y $16,7 \%$ de los pacientes que fueron valorado en niveles bajos, $23,1 \%$ de los pacientes en niveles medios y el $34,6 \%$ y $23,1 \%$ respectivamente en niveles medios alto y alto en los indicadores relacionados con el cumplimiento.

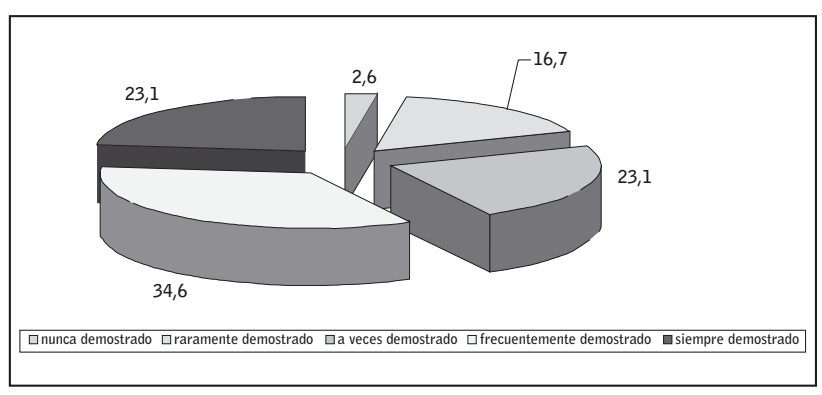

Gráfico 2: Indicadores relacionados con el criterio de resultado. Conducta de cumplimiento

El gráfico 3 presenta la frecuencia relativa de los indicadores relacionados con los criterios de resultado control del riesgo, distribuyéndose de la siguiente manera: el 2,6\% y $19,2 \%$ de los pacientes fueron valorados en niveles bajos, el $29,5 \%$ en niveles medios, el $42,3 \%$ de los pacientes en niveles altos y el $6,3 \%$ fueron valorados en niveles muy altos en los indicadores relacionados con el control del riesgo.

El gráfico 4 presenta la frecuencia relativa de los indicadores relacionados con los criterios de resultado control de síntomas, observándose que solo el $8 \%$ de los pacientes fueron valorados en niveles bajos, mientras aproximadamente el $67 \%$ de los pacientes fueron valorados en nivele altos y muy altos en los indicadores relacionados con el de control de síntomas. 


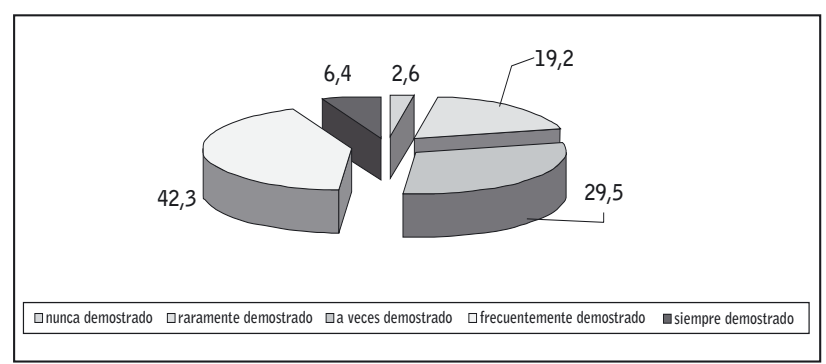

Gráfico 3 : Indicadores relacionados con el criterio de resultado. Control de riesgo

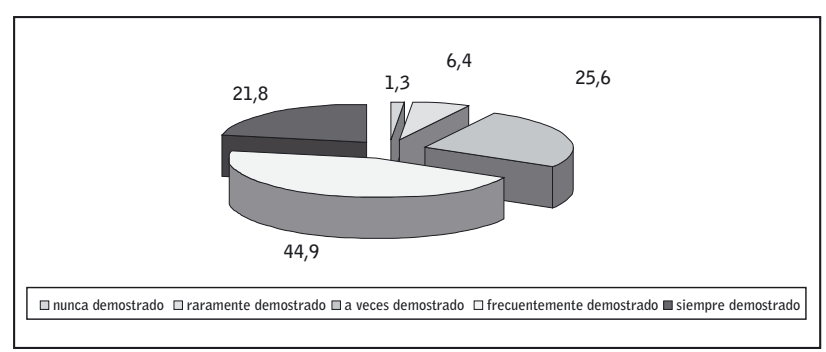

Gráfico 4: Indicadores relacionados con el criterio de resultado. Control de síntomas

La tabla 1 presenta los indicadores relacionados con el conocimiento del manejo del régimen terapéutico, en relación con el nivel de conocimientos adquiridos en el entrenamiento. Observándose como estos indicadores, han mejorado generalmente en todos los pacientes, en relación con los conocimientos adquiridos durante el entrenamiento. Los indicadores medidos han sido: describe la dieta, describe la medicación y su uso, describe los procedimientos terapéuticos, describe las complicaciones potenciales, describe signos y síntomas de las complicaciones.

Hemos destacado el \% de pacientes cuya valoración ha bajado en relación a los conocimientos adquiridos durante el entrenamiento.

La tabla 2 refleja los indicadores relacionados con el criterio de resultado conducta de cumplimiento en relación con el nivel de conocimientos adquiridos en el entrenamiento, observándose que un $\mathrm{n}^{\circ}$ importante de nuestros pacientes no alcanzan niveles de cumplimiento adecuados a los conocimientos adquiridos durante el entrenamiento. Los indicadores medidos ha sido los siguientes: comunica seguir pautas descritas, confianza en el profesional sanitario sobre la información recibida, conserva la cita, modifica la pauta orientada por el profesional sanitario, realiza las actividades de la viada diaria según prescripción Destaca el porcentaje de pacientes que habiendo obtenido un nivel de conocimientos entre moderado y sustancial durante el entrenamiento, mantienen un nivel de cumplimiento entre nunca demostrado y raramente demostrado.

\begin{tabular}{|l|l|l|l|l|l|}
\hline Evaluación entrenamiento & ninguno & escaso & moderado & sustancial & extenso \\
\hline Ninguno & 0 & 0 & 0 & 0 & 0 \\
\hline Escaso & 0 & 0 & $5(45,5 \%)$ & $3(27,3 \%)$ & $3(27,3 \%)$ \\
\hline Moderado & 0 & 0 & $4(15,6 \%)$ & $13(40,6 \%)$ & $14(50,3 \%)$ \\
\hline Sustancial & 0 & 0 & 0 & $11(34,4 \%)$ & $21(65,6 \%)$ \\
\hline Extenso & 0 & 0 & 0 & 0 & $3(100 \%)$ \\
\hline
\end{tabular}

Tabla 1: Evolución de los conocimientos del manejo del régimen terapéutico según nivel de conocimientos al alta

\begin{tabular}{|l|l|l|l|l|l|}
\hline Evaluación entrenamiento & Nunca demostrado & $\begin{array}{l}\text { Raramente } \\
\text { demostrado }\end{array}$ & $\begin{array}{l}\text { A veces } \\
\text { demostrado }\end{array}$ & $\begin{array}{l}\text { Frecuentemente } \\
\text { demostrado }\end{array}$ \\
\hline Ninguno (menor de 31\%) & 0 & 0 & 0 & 0 & 0 \\
\hline Escaso (entre 31 y $50 \%)$ & 0 & $5(45,5 \%)$ & $4(36,4 \%)$ & $2(18,2 \%)$ & 0 \\
\hline Moderado (entre 51 y $70 \%)$ & $2(6,3 \%)$ & $6(18,8 \%)$ & $7(21,9 \%)$ & $9(28,1 \%)$ & $8(25 \%)$ \\
\hline Sustancial (entre 71 y $90 \%)$ & 0 & $2(6,3 \%)$ & $7(21,9 \%)$ & $14(43,8 \%)$ & $9(28,1 \%)$ \\
\hline Extenso (mayor de $90 \%)$ & 0 & 0 & 0 & $2(66,7 \%)$ & $1(33,3 \%)$ \\
\hline
\end{tabular}

Tabla 2: Conducta de cumplimiento relacionado con los conocimientos al alta 
La tabla 3 presenta los indicadores relacionados con el criterio de resultado control del riesgo: modifica estilo de vida, reconoce factores de riesgo, reconoce cambios en su estado de salud, participa en la identificación sistemática de los riesgos identificados y desarrolla estrategias de control de riesgos, en los distintos niveles de evaluación durante el entrenamiento, donde destaca el hecho de que un porcentaje importante de pacientes tengan los indicadores peor valorados, especialmente entre los que obtuvieron niveles de conocimientos entre moderado y sustancial

La tabla 4 presenta los indicadores relacionados con el control de síntomas en los distintos niveles de conocimientos adquiridos durante el entrenamiento habiéndose medidos los siguientes los indicadores: reconoce síntomas de sobre hidratación, reconoce síntomas de infección, utiliza signos de alarmas para buscar atención sanitaria, utiliza los recursos disponibles, utiliza medidas preventivas. Desatacando el hecho de que estén mas bajos estos indicadores sobre todo en los pacientes con conocimientos medios o medios altos al alta de entrenamiento.

\section{Discusión}

El conocimiento sobre el régimen terapéutico de nuestros pacientes aumenta de una manera significativa en valoraciones posteriores, en relación al nivel de conocimientos adquiridos en el momento del alta de entrenamiento que coincide con el alta a domicilio. Posiblemente se deba al refuerzo continuado que supone el seguimiento enfermero de estos pacientes, tanto en el domicilio mediante contacto telefónico o visita domiciliaria como en las revisiones hospitalarias.

Los indicadores relacionados con el criterio de resultado conducta de cumplimiento en nuestros pacientes aparece con valoraciones bajas o medio bajas en el $18 \%$ de ellos y nos llama la atención que pacientes que obtuvieron valoraciones altas en conocimientos han bajado el nivel de cumplimiento desde el inicio del tratamiento, coincidiendo con otros autores en lo que Ilaman "relajación de las pautas indicadas"3.

El control del riesgo medido a través de indicadores relacionados también aparece con valoraciones bajas o medio bajas en el 19,4\% de los pacientes, algunos de ellos que habían obtenido mejores valoraciones al alta de entrenamiento.

En el criterio de resultados control de síntomas, las valoraciones bajas o muy bajas aparecen solo en el $5,5 \%$ de los pacientes, lo que indica que la mayoría de los pacientes reconocen los síntomas de las complicaciones y actúan en consecuencia según indicaciones pautadas.

\begin{tabular}{|l|l|l|l|l|l|}
\hline Evaluación entrenamiento & Nunca demostrado & $\begin{array}{l}\text { Raramente } \\
\text { demostrado }\end{array}$ & $\begin{array}{l}\text { A veces } \\
\text { demostrado }\end{array}$ & $\begin{array}{l}\text { Frecuentemente } \\
\text { demostrado }\end{array}$ & $\begin{array}{l}\text { Siempre } \\
\text { demostrado }\end{array}$ \\
\hline Ninguno (menor de 31\%) & 0 & 0 & 0 & 0 & 0 \\
\hline Escaso (entre 31 y 50\%) & $1(9,1 \%)$ & $5(45,5 \%)$ & $4(36,4 \%)$ & $1(9,1 \%)$ & 0 \\
\hline Moderado (entre 51 y 70\%) & $1(3,1 \%)$ & $9(28,1 \%)$ & $10(31,3 \%)$ & $11(39,4 \%)$ & $1(3,1 \%)$ \\
\hline Sustancial (entre 71 y 90\%) & 0 & $1(3,1 \%)$ & $9(28,1 \%)$ & $19(59,4 \%)$ & $3(9,4 \%)$ \\
\hline Extenso (mayor de 90\%) & 0 & 0 & 0 & $2(66,7 \%)$ & $1(33,3 \%)$ \\
\hline
\end{tabular}

Tabla 3: Control del riesgo relacionado con los conocimientos al alta

\begin{tabular}{|l|l|l|l|l|l|}
\hline Evaluación entrenamiento & Nunca demostrado & $\begin{array}{l}\text { Raramente } \\
\text { demostrado }\end{array}$ & $\begin{array}{l}\text { A veces } \\
\text { demostrado }\end{array}$ & $\begin{array}{l}\text { Frecuentemente } \\
\text { demostrado }\end{array}$ & $\begin{array}{l}\text { Siempre } \\
\text { demostrado }\end{array}$ \\
\hline Ninguno (menor de 31\%) & 0 & 0 & 0 & 0 & 0 \\
\hline Escaso (entre 31 y 50\%) & $1(9,1 \%)$ & $2(18,2 \%)$ & $3(27,3 \%)$ & $4(36,4 \%)$ & $1(9,1 \%)$ \\
\hline Moderado (entre 51 y 70\%) & 0 & $3(9,4 \%)$ & $10(31,3 \%)$ & $14(43,8 \%)$ & $5(15,6 \%)$ \\
\hline Sustancial (entre 71 y 90\%) & 0 & 0 & $7(21,9 \%)$ & $16(50 \%)$ & $9(28,1 \%)$ \\
\hline Extenso (mayor de 90\%) & 0 & 0 & 0 & $1(33,3 \%)$ & $2(66,7 \%)$ \\
\hline
\end{tabular}

Tabla 4: Control de síntomas relacionados con los conocimientos al alta 
De acuerdo con los resultados obtenidos en nuestro estudio, no consideramos la necesidad de establecer reentrenamiento normalizado, en relación a los conocimientos obtenidos y en los que permanecen en el tiempo que en todos nuestros pacientes han mejorado, posiblemente condicionado al hecho de que estos conocimientos sean continuamente reforzados en las visitas como parte de la continuidad del cuidado enfermero, sobre todo en los pacientes con valoraciones mas bajas, a lo que ayuda conocer la evaluación al alta ${ }^{8}$.

Así mismo consideramos que se deben establecer pautas de actuación enfermera que refuercen en continuidad, las conductas de cumplimiento, para poder evitar complicaciones especialmente las relacionadas con la infección y la sobrehidratación, como puede ser la negociación con el paciente y la utilización del paciente como agente decisor razonado. La mayoría de estudios consiguen mejorar el cumplimiento entre el 5 y el 10 $\%$, aunque nos encontremos algún estudio que consigue hasta un $35 \%$ de mejora. La mejor estrategia es una combinación de diferentes estrategias, más que alguna de forma individual ${ }^{9}$.

Recibido: Enero 2011

Revisado: Febrero 2011

Modificado: Abril 2011

Aceptado: Mayo 2011

\section{Bibliografia:}

1. Tejuca Marenco M. "Adiestramiento de los pacientes en DPA" - Tercer Curso andaluz de diálisis peritoneal para enfermería. SEDEN Madrid 2001; Pág. 169176.

2. Granado A., Ruiz C., Arrieta J. Adiestramiento del paciente que se incorpora a diálisis peritoneal. Guías de práctica clínica en diálisis peritoneal SEN.www. senefro.org. pag 108-122.

3. Castro MJ., Sánchez S., Celadilla 0., Martínez M.V. Enseñanza de las técnicas dialíticas peritoneales. En Montenegro J., Olivares J. La diálisis peritoneal. Dibe S.L. España1999. pp. 181-199.

4. Martín Espejo JL., Gómez Castilla AC. Enseñanza en diálisis peritoneal contínua ambulatoria (DPCA). En Manual práctico de diálisis peritoneal SEN-SEDEN. Atrium comunicación estratégica S.L. Badalona 2005 Pág. 299-305.

5. Gómez C., Sánchez J., Ojeda A."Programa de entrenamiento de diálisis peritoneal. La visita domiciliaria. Experiencia de 9 años". Rev Soc Esp Enferm Nefrol 2000; 3 (1): 6/13.

6. Portela Rico I., Pérez López T., Bermúdez Liste C., Morrondo Remis Salgado Silva D. Enseñanza en diálisis peritoneal automática (DPA). En Manual práctico de diálisis peritoneal SEN-SEDEN. Atrium comunicación estratégica S.L. Badalona 2005 Pág. 307-312.

7. Martín Espejo J. L., - Cirera Segura F., Reina Neyra M. Formación proporcionada a los pacientes de diálisis peritoneal. Rev Soc Esp Enferm Nefrol 2008; 11 (1):13/19.

8. Gómez Castilla A.C., Ojeda Guerrero M.A., Los indicadores sobre conocimiento de la infección como predictor de la morbilidad infecciosa en pacientes de diálisis peritoneal. En Libro de comunicaciones del XXXIII congreso nacional de la SEDEN. Hospal 2008. P. 357-362.

9. Márquez Contreras E, Casado Martínez JJ, Márquez Cabeza JJ. Estrategias para mejorar el cumplimiento. Formación Medica Continuada Atención Primaria Elsevier.es 2001; 8:558-573.

10. Gruart Armangue P. Reentrenamientos a pacientes en diálisis peritoneal. En Manual práctico de diálisis peritoneal SEN SEDEN. Atrium comunicación estratégica S.L. Badalona 2005.

11. Clasificación de Intervenciones de Enfermería. (NIC) J.C. McCloskay y G.M. Bulechek. Madrid. Harcourt. Mosby. Elselvier 2002.

12. Clasificación de Resultados de Enfermería. (NOC) S. Moorhead, M Johnson, M. Maas Bulechek. Madrid. Harcourt. Mosby. Elselvier 2002. 